\title{
Palmocervical reflex
}

\section{A hyperactive palmomental reflex?}

吕

An 85-year-old, right-handed, Caucasian man with a 14-year history of idiopathic Parkinson disease presented with progressive cognitive decline. An involuntary contraction of ipsilateral mentalis and platysma muscles was observed while stroking the patient's left thenar eminence. The common palmomental reflex was present on the right side (video on the Neurology ${ }^{\circledR}$ Web site at www.neurology.org).

The palmomental reflex is an involuntary contraction of the mentalis muscle with the stimulation of the thenar eminence. Its presence suggests the possibility of cerebral pathology. ${ }^{1}$ Involvement of the platysma muscle may result from an activation of neighboring motor neuron pools comparable to pathologic spread. ${ }^{2}$ This can be considered as palmomental reflex hyperactivity.

\section{Chakorn Chansakul, MD, Mohamed Teleb, MD, Roberto Bomprezzi, MD, PhD, Phoenix, AZ}

Disclosure: Dr. Chansakul and Dr. Teleb report no disclosures. Dr. Bomprezzi serves as PI for clinical trials supported by Genzyme Corporation, Novartis, Merck Serono, Biogen Idec, Genentech, Inc., and UCB; and received a 1-year clinical fellowship funded by the National Multiple Sclerosis Society.

Address correspondence and reprint requests to Dr. Chakorn Chansakul, Barrow Neurological Institute, 350 West Thomas Road, Phoenix, AZ 85013; Chakorn.Chansakul@chw.edu

Supplemental data at www.neurology.org
1. Owen G, Mulley GP. The palmomental reflex: a useful clinical sign? J Neurol Neurosurg Psychiatry 2002;73:113-115.

2. Dick JP. The deep tendon and the abdominal reflexes. J Neurol Neurosurg Psychiatry 2003;74:150-153. 


\title{
Neurology
}

\author{
Palmocervical reflex: A hyperactive palmomental reflex? \\ Chakorn Chansakul, Mohamed Teleb and Roberto Bomprezzi \\ Neurology 2010;74;91 \\ DOI 10.1212/WNL.0b013e3181c7daa3
}

This information is current as of December 28, 2009

\begin{tabular}{|c|c|}
\hline $\begin{array}{l}\text { Updated Information \& } \\
\text { Services }\end{array}$ & $\begin{array}{l}\text { including high resolution figures, can be found at: } \\
\text { http://n.neurology.org/content/74/1/91.full }\end{array}$ \\
\hline Supplementary Material & $\begin{array}{l}\text { Supplementary material can be found at: } \\
\text { http://n.neurology.org/content/suppl/2009/12/27/74.1.91.DC1 }\end{array}$ \\
\hline References & $\begin{array}{l}\text { This article cites } 2 \text { articles, } 2 \text { of which you can access for free at: } \\
\text { http://n.neurology.org/content/74/1/91.full\#ref-list-1 }\end{array}$ \\
\hline Subspecialty Collections & $\begin{array}{l}\text { This article, along with others on similar topics, appears in the } \\
\text { following collection(s): } \\
\text { All Cognitive Disorders/Dementia } \\
\text { http://n.neurology.org/cgi/collection/all_cognitive_disorders_dementia } \\
\text { Assessment of cognitive disorders/dementia } \\
\text { http://n.neurology.org/cgi/collection/assessment_of_cognitive_disorde } \\
\text { s_dementia } \\
\text { Clinical neurology examination } \\
\text { http://n.neurology.org/cgi/collection/clinical_neurology_examination }\end{array}$ \\
\hline Permissions \& Licensing & $\begin{array}{l}\text { Information about reproducing this article in parts (figures,tables) or in } \\
\text { its entirety can be found online at: } \\
\text { http://www.neurology.org/about/about_the_journal\#permissions }\end{array}$ \\
\hline Reprints & $\begin{array}{l}\text { Information about ordering reprints can be found online: } \\
\text { http://n.neurology.org/subscribers/advertise }\end{array}$ \\
\hline
\end{tabular}

Neurology ${ }^{\circledR}$ is the official journal of the American Academy of Neurology. Published continuously since 1951, it is now a weekly with 48 issues per year. Copyright . All rights reserved. Print ISSN: 0028-3878. Online ISSN: 1526-632X.

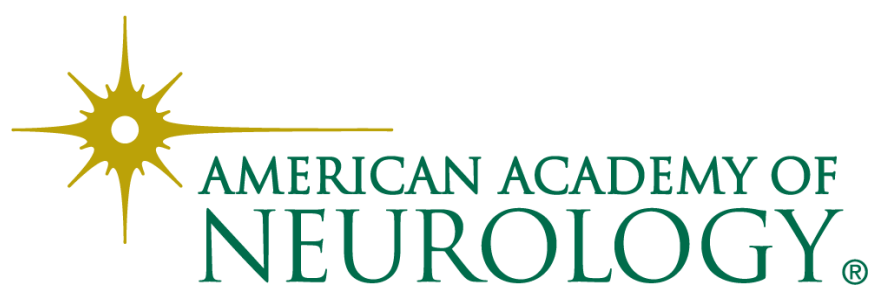

